\title{
On Curvatures of Homogeneous Finsler Space with Special $(\alpha, \beta)$-Metric
}

\author{
Chandru K. and Narasimhamurthy S.K. \\ Department of Mathematics, Kuvempu University, Jnana Sahyadri, Shankaraghatta-577 451, Shimoga, \\ Karnataka, INDIA.
}

\begin{abstract}
In this paper, we give an explicit formula of the S-curvature of homogeneous Finsler space with special $(\alpha, \beta)$-metric and proved that a homogeneous Finsler space with almost isotropic $S$-curvature must have vanishing $S$-curvature. We also derived an explicit formula of the mean-Berwald curvature $E_{i j}$ of homogeneous Finsler space.
\end{abstract}

Keywords: Finsler space, $(\alpha, \beta)$-metric, Homogeneous Finsler space, S-curvature, Mean-Berwald curvature.

\section{Introduction}

Let $(M, F)$ be a Finsler space, where $M$ be the connected smooth manifold and $F$ be the Finsler metric. $(\alpha, \beta)$-metrics are the class of Finsler metrics, for example Randers metric, Kropina metric, Matsumoto metric, etc. $(\alpha, \beta)$-metrics are of the form $F=\phi(s)$, where $s=\frac{\beta}{\alpha}$, here $\alpha$ is Riemannian metric and $\beta$ is the one form. One of the central problems in Finsler geometry is to study curvature properties of special class of Finsler spaces such as homogeneous-Finsler spaces with $(\alpha, \beta)$-metrics.

A Finsler space $(M, F)$ becomes homogeneous if the group of isometries $I(M, F)$ of $(M, F)$ acts transitively on $M$. As described in [7], for homogeneous Finsler space, $M$ can be written as a coset space $G / H$ with $(\alpha, \beta)$ metric of the form $F=\phi(s)$, where $s=\frac{\beta}{\alpha}$, with $\alpha$ a $G$ invariant Riemannian metric on $G / H$ and $\beta$ a $G$-invariant vector field on $G / H$. Therefore the Lie algebra g of $G$ expressed as composition of $h$ and $m$.

$$
g=h+m \text { (direct sum of subspaces) }
$$

such that $A d(h)(m) \subset m, h \in H$, and we can identify $M$ with the tangent space of $(G / H)$ at the origin $o=H$. Further, the one form $\beta$ corresponds to a vector field $\bar{X}$ on $G / H$ which is generated by $\operatorname{Ad}(H)$-invariant vector in $m$ with length $<1$.

The goal of this paper is to derive an explicit formula for the S-curvature of homogeneous Finsler space with special $(\alpha, \beta)$-metric $F=\frac{\alpha^{2}}{\beta}+\beta$.The notion of S-curvature for a Finsler space introduced by Z. Shen in [14]. It is a quantity to measure the rate of change of the volume form of a Finsler space along the geodesics. Recently many geometers studied curvature properties of homogeneous Finsler space [7, 8]. In 2007, S. Deng and Z. Hou studied that a homogeneous Finsler spaces with non-positive flag curvature [8]. In 2010, S. Deng obtained the explicit formula of S-curvature of homogeneous Randers space with almost isotropic S-curvature must have vanishing S-curvature [7]. As for reference, there is an explicit formula for S-curvature in a local standard coordinate system by Z. Shen [14]. However, for a homogeneous spaces there should have a formula which does not use local coordinates. In this article we are studied curvature properties of homogeneous Finsler space with special $(\alpha, \beta)$-metric $F=\frac{\alpha^{2}}{\beta}+\beta$ and find the formula for S-curvature and mean-Berwald curvature.

In the following, we shall use Einstein summation convention unless otherwise stated.

\section{Preliminaries}

Finsler space is a smooth manifold possessing a Finsler metric. A standard definition of a Finsler space is defined by:

Definition 2.1. A Finsler space is a triple $F^{n}=(M, D, F)$, where $M$ is an n-dimensional manifold, $D$ is an open subset of a tangent bundle TM and $F$ is a Finsler metric defined as a function $F: T M \rightarrow[0,1)$, with the following properties:

1. Regular: $F$ is $C^{1}$ on the entire tangent bundle $T M \backslash\{0\}$.

2. Positive homogeneous: $F(x, \lambda y)=\lambda F(x, y)$.

3. Strong convexity: The $n \times n$ Hessian matrix

$$
g_{i j}=\left[F^{2}\right]_{y^{i} y^{j}}
$$

is positive definite at every point on $T M \backslash\{0\}$, where $T M \backslash\{0\}$ denotes the tangent vector $y$ is non zero in the tangent bundle TM. 
In Finsler geometry, important class of Finsler metrics is $(\alpha, \beta)$-metrics [9]. A Finsler metric $F=F(x, y)$ is said to be $(\alpha, \beta)$--metric if it can be expressed in the form,

$$
F=\phi(s), s=\frac{\beta}{\alpha}
$$

where $\alpha=\sqrt{a_{i j}(x) y^{i} y^{j}}$ is a Riemannian metric and $\beta=b_{i}(x) y^{i}$ is a 1 -form. Here $\phi=\phi(s)$ is a $C^{1}$ positive function on an open interval $\left(-b_{0}, b_{0}\right)$ satisfying,

$$
\phi(s)-s \phi^{\prime}(s)+\left(b^{2}-s^{2}\right) \phi^{\prime \prime}(s)>0, \quad|s| \leq 0<b 0, \quad b=|| \beta(x) \|_{\alpha}
$$

In this article we considered an important example of such $(\alpha, \beta)$-metrics is $F=\frac{\alpha^{2}}{\beta}+\beta$, expressed in the following form,

$$
\begin{aligned}
F & =\alpha(x, y)\left\{\frac{\alpha(x, y)}{\beta(x y)}+\frac{\beta(x, y)}{\alpha(x y)}\right\} \\
& =\alpha \phi(s) ; \phi(s)=\frac{1}{s}+s,
\end{aligned}
$$

where $s=\frac{\beta}{\alpha}, \alpha=\sqrt{a_{i j}(x) y^{i} y^{j}}$ is the (2.3) and (2.4) Riemannian metric and $\beta=b_{i}(x) y^{i}$ is the 1 -form.

Let

$$
r^{i j}=\frac{1}{2}\left(b_{i \mid j}+b_{j \mid i}\right), \quad s_{i j}=\frac{1}{2}\left(b_{i \mid j}-b_{j \mid i}\right) .
$$

Clearly, $\beta$ is closed if and only if $s_{i j}=0$. We put

$$
\begin{aligned}
& r_{i 0}=r_{i j} y^{j}, \quad r_{00}=r_{i j} y^{i} y^{j}, \quad r_{j}=b^{i} r_{i j}, \\
& s_{i 0}=s_{i j} y^{j}, \quad s_{j}=b^{i} s_{i j}, \quad r_{0}=r_{j} y^{j}, s_{0}=s_{j} y^{j}
\end{aligned}
$$

For a Finsler metric $F=F(x, y)$, the geodesics are characterized by system of second order ordinary differential equations,

$$
\ddot{x}^{i}+2 G^{i}(x, \dot{x})=0,
$$

where $G^{i}=G^{i}(x, y)$ are called spray coefficients and are given by,

$$
G^{i}=y^{i} \frac{\partial}{\partial x^{i}}-2 G i(x, y) \frac{\partial}{\partial x^{i}}
$$

in standard local coordinates $\left(x^{i}, y^{i}\right)$ in $T M$, then the spray of $F$ is the vector field $G^{i}=y^{i} \frac{\partial}{\partial x^{i}}-$ $2 \operatorname{Gi}(x, y) \frac{\partial}{\partial x^{i}}[9]$.

The S-curvature is an important non-Riemannian quantity introduced by Z. Shen [14], defined with respect to a volume form $\mathrm{dV}=\_(\mathrm{x}) \mathrm{dx}$ by,

$$
S=\frac{\partial G^{m}}{\partial y^{m}}-y^{m} \frac{\partial}{\partial x^{m}}\left(\ln \sigma_{F}(x)\right)
$$

Here the volume form can be the Busemann-Housdorff volume form $d V_{B H}=\sigma_{B H}(x) d x$, where

$$
\sigma_{B H}(x)=\frac{\operatorname{Vol}\left(B^{n}\right)}{\operatorname{Vol}\left\{\left(y^{i}\right) \in R^{n} \mid F(x, y)<1\right\}}
$$

or the Holmes-Thompson volume form $d V_{H T}=\sigma_{H T}(x) d x$, where

$$
\sigma_{H T}(x)=\frac{1}{\operatorname{Vol}\left(B^{n}\right)} \int_{F(x, y)<1} \operatorname{det}\left(g_{i j}(x, y) d y\right) .
$$

Unless specified, the S-curvature usually defined with respect to the Busemann-Housdorff volume form.

Definition 2.2. A Finsler space $(M, F)$ is said to have almost isotropic $S$-curvature if there exists a smooth function $c(x)$ on $M$ and a closed 1 -form $\eta$ such that:

$$
S(x, y)=(n+1) c(x) F(y)+\eta(y), \quad x \in M, \quad y \in T_{x} M .
$$

Note: (1). $F$ is of isotropic S-curvature if $c=c(x)$ is a scalar for and $\eta=0$.

(2). $F$ is of constant S-curvature if $\mathrm{c}$ is a constant and $\eta=0$.

In [12], authors defined the S-curvature of the $(\alpha, \beta)$--metric $F=\phi(s)$, in local coordinate system by,

where

$$
S=\left\{2 \psi-\frac{f^{\prime}(b)}{b f(b)}\right\}\left(r_{0}+s_{0}\right)-\alpha^{-1} \frac{\Phi}{2 \Delta^{2}}\left(r_{00}-2 \alpha Q s_{0},\right.
$$

$$
\begin{aligned}
& Q=\frac{2+2 s}{1-s^{2}}, \quad \Delta=1+s Q+\left(b^{2}-s^{2}\right) Q^{\prime}, \quad \psi=Q^{\prime} / 2 \Delta \\
& \Phi=-\left(Q-s Q^{\prime}\right)\{n \Delta+1+s Q\}-\left(b^{2}-s^{2}\right)(1+s Q) Q^{\prime \prime}
\end{aligned}
$$

and the function $f(b)$ in the formula is defined as follows, 


$$
f(b)= \begin{cases}\frac{\int_{0}^{\pi} \sin ^{\mathrm{n}-2} t d t}{\int_{0}^{\pi} \frac{\sin ^{\mathrm{n}-2} \mathrm{t}}{\phi(\mathrm{b} \cos \mathrm{t})^{\mathrm{n}}} d t} & \text { if } d V=d V_{B H} \\ \frac{\int_{0}^{\pi}\left(\sin ^{\mathrm{n}-2} \mathrm{t}\right) T(\mathrm{~b} \cos \mathrm{t}) d t}{\int_{0}^{\pi} \sin ^{\mathrm{n}-2} t d t} & \text { if } d V=d V_{-} H T\end{cases}
$$

where $T(s)=\phi\left(\phi-s \phi^{\prime}\right)^{n-2}\left\{\left(\phi-s \phi^{\prime}\right)+\left(b^{2}-s^{2}\right) \phi^{\prime \prime}\right\}$. Then the volume form $d V$ is given by $d V=$ $f(b) d V_{\alpha}$, where $d V=\sqrt{\operatorname{det}\left(\alpha_{i j}\right) d x}$, denote the Riemannian volume form of $\alpha$.

\section{S-Curvature Of Homogeneous Finsler Space}

Two Finsler spaces $\left(M_{1}, F_{1}\right)$ and $\left(M_{2}, F_{2}\right)$ are said to be isometric if there exists a diffeomorphism $\phi$ from $M_{1}$ onto $M_{2}$, such that $F_{1}(x, y)=F_{2}(\phi(x), \phi(y))$ for any $x \in M_{1}$ and $y \in T_{x} M_{1}$. In 2006 [6], S. Deng and Z. Hou proved that the group of isometrics of Finsler space is a Lie transformation group on the original manifold which can be used to study homogeneous Finsler spaces.

A homogeneous Finsler space $M$ can be written as a coset space $G / H$ with a $G$-invariant $(\alpha, \beta)$-metric $F=\frac{\alpha^{2}}{\beta}+$ $\beta$, where both Riemannian metric $\alpha$ and the 1-form $\beta$ are invariant under the action of $G$. In [8], S. Deng and Z. Hou, specified that, $\beta$ corresponding to a unique vector $u$ in $T_{o}(G / H)$ which is fixed under linear isotropy representation of $H$ on $T_{o}(G / H)$ and $o=H$ is the origin of $G / H$. And in [7], S. Deng and X. Wang gives the following result,

Theorem 3.1.[7] Consider an G-invariant $(\alpha, \beta)$-metric $F=\phi(s)$ on the reduction homogeneous manifold $G / H$ with a decomposition of the Lie algebra

Then the $S$-curvature of $F$ has the form

$$
g=h+m
$$

$$
S(o, y)=-\frac{1}{\alpha(y)} \frac{\Phi}{2 \Delta^{2}}\{-c\langle[u, y] m, y\rangle-\alpha(y) Q\langle[u, y] m, u\rangle\}, y \in m,
$$

where $u$ is the vector in $m$ corresponding to the 1-form $\beta$, and we have identified $m$ with the tangent space of $G / H$ at origin $O=H$.

According to the above theorem (3.1), in this section we find the formula of $S$-curvature for homogeneous Finsler space with special $(\alpha, \beta)$-metric. By definition (2.1), we can write $(\alpha, \beta)$-metric $F=\frac{\alpha^{2}}{\beta}+\beta$ as $F=$ $\phi(s)$, where $\phi(s)=\frac{1}{s}+s$. Now, by the equations (2.15) and (2.16) we obtained,

$$
\begin{aligned}
& Q=\frac{s^{2}-1}{2 s}, \quad Q^{\prime}=\frac{s^{2}+1}{2 s^{2}}, \\
& \Delta=\frac{b^{2}\left(s^{2}+1\right)}{2 s^{2}}, \quad \Phi=\frac{(n+1) b^{2}\left(1+s^{2}\right)}{2 s^{3}} .
\end{aligned}
$$

Since $(G / H, F)$ is homogeneous, now we have to compute the $S$-curvature at the origin $o=H$. Let $\left(U,\left(x^{1}, x^{2}, \ldots, x^{n}\right)\right)$ be the local coordinate system. With reference to [5], to find the S-curvature in local coordinate system, we need to evaluate the following quantities at the origin:

1. $r_{i j}=\frac{1}{2}\left(b_{i ; j}+b_{j ; i}\right)$ and $b_{i}$ 's are defined by $\beta=b_{i}(x) d x^{i}$,

2. $s_{i}=b_{j} s_{i}^{j}$ and $s_{j}^{i}$ are defined by $s_{j}^{i}=\frac{1}{2}\left(\frac{\partial b_{i}}{\partial x^{j}}-\frac{\partial b_{j}}{\partial x^{i}}\right)$,

3. $s_{0}=s_{i} y^{i}$,

4. $\rho_{0}=\rho_{x^{i}} y^{i}$, where $\rho=\sqrt{1-|| \beta||}$ and $\|\beta\|$ is the length of the 1 -form $\beta$ with respect to $\alpha$.

Let $<,>$ be the inner product on $m$ and consider the Levi-Civita connection of $(G / H, \alpha)$ which will be useful to calculate the S-curvature of homogeneous Finsler spaces. Then for $v \in g$, define a one parameter transformation group $\phi_{t}, t \in R$ of $G / H$ by

$$
\phi_{t}(g H)=(\exp (t v) g) H, \quad g \in G .
$$

Now $\phi_{t}$ generates a vector field on $G / H$ which is a Killing vector field (this is called the fundamental vector field generated by $\mathrm{v}$ in [8]) and we denote this vector field by $\bar{v}$. In [12] authors defined the following formula,

$$
\left\langle\left.\nabla_{v_{1}} v_{2}\right|_{o}, w\right\rangle=\frac{1}{2}\left(-\left\langle\left[v_{1}, v_{2}\right]_{m}, w\right\rangle+\left\langle\left[w, v_{2}\right]_{m}, v_{1}\right\rangle+\left\langle\left[w, v_{1}\right]_{m}, v_{2}\right\rangle\right),
$$

where $v_{1}, v_{2}, w \in m, o=H$ is the origin of the coset space and $\left[v_{1}, v_{2}\right]_{m}$ denote the projection of $\left[v_{1}, v_{2}\right]$ to $m$ corresponding to the decomposition (3.1).

Now by using the formula (2.5), we have to compute $r_{00}$ and $s_{0}$. First consider,

and by differentiating $b_{i}$ we get,

$$
b_{i}=\beta\left(\frac{\partial}{\partial x^{i}}\right)=\left\langle\bar{u}, \frac{\partial}{\partial x^{i}}\right\rangle=c\left\langle\frac{\partial}{\partial x^{n}}, \frac{\partial}{\partial x^{i}}\right\rangle,
$$




$$
\begin{aligned}
\frac{\partial b_{i}}{\partial x^{j}} & =c \frac{\partial}{\partial x^{j}}\left\langle\frac{\partial}{\partial x^{n}}, \frac{\partial}{\partial x^{i}}\right\rangle \\
& =c\left(\left\langle\nabla_{\frac{\partial}{\partial x^{j}}} \frac{\partial}{\partial x^{n}}, \frac{\partial}{\partial x^{i}}\right\rangle+\left\langle\frac{\partial}{\partial x^{n}}, \nabla_{\frac{\partial}{\partial x^{j}}} \frac{\partial}{\partial x^{i}}\right\rangle\right) .
\end{aligned}
$$

Hence at the origin we have (here we use the symmetry of the connection $\nabla_{\frac{\partial}{\partial x^{j}}} \frac{\partial}{\partial x^{i}}-\nabla_{\frac{\partial}{\partial x^{i}}} \frac{\partial}{\partial x^{j}}-\left[\frac{\partial}{\partial x^{i}}, \frac{\partial}{\partial x^{j}}\right]=$ $0)$,

$$
s_{i j}(o)=\frac{1}{2} c c\left(\left\langle\nabla_{\frac{\partial}{\partial x^{n}}} \frac{\partial}{\partial x^{j}}, \frac{\partial}{\partial x^{i}}\right\rangle+\left\langle\frac{\partial}{\partial x^{n}}, \nabla_{\frac{\partial}{\partial x^{i}}} \frac{\partial}{\partial x^{j}}\right\rangle\right)
$$

Then by equation (3.6) we have,

$$
s_{i j}(o)=\frac{1}{2}\left\langle\left[u_{i}, u_{j}\right]_{m}, u_{n}\right\rangle
$$

Since at the origin we have $\left(a_{i j}\right)=I_{n}$, we get

Hence

$$
s_{j}^{i}(o)=a^{i k}(o) s_{k j}(o)=\sum_{k-1}^{n} \delta_{i}^{k} s_{k j}(o)=s_{i j}(o) .
$$

Now for $y=y^{i} u_{i} \in m$, we have

$$
s_{i}(o)=b_{l}(o) s_{i}^{l}(o)=c s_{i}^{n}(o)=s_{n i}(o) .
$$

$$
\begin{aligned}
s_{0}(y) & =y^{l} s_{l}(o)=c y^{l} s_{n l}(o)=\frac{1}{2} y^{l}\left\langle\left[u_{n}, u_{l}\right]_{m}, u_{n}\right\rangle \\
& =\frac{1}{2}\left\langle\left[c u_{n}, y^{l} u_{l}\right]_{m}, \quad c u_{n}\right\rangle \\
& =\frac{1}{2}\left\langle[u, y]_{m}, u_{i}\right\rangle .
\end{aligned}
$$

Next we compute $r_{i j}$. Suppose $i \geq j$. Then we have

$$
\begin{aligned}
r_{i j}(o) & =\left.\frac{1}{2}\left(b_{i ; j}+b_{j ; i}\right)\right|_{o} \\
& =\frac{1}{2}\left(\frac{\partial b_{i}}{\partial x^{j}}-b_{l} \Gamma_{j i}^{l}+\frac{\partial b_{j}}{\partial x^{i}}-\left.b_{l} \Gamma_{j i}^{l}\right|_{o}\right. \\
& =\left.\frac{1}{2}\left(\frac{\partial b_{i}}{\partial x^{j}}+\frac{\partial b_{j}}{\partial x^{i}}\right)\right|_{o}-c \Gamma_{j i}^{l}(o) .
\end{aligned}
$$

By (3.8) we have

$$
\left.\frac{1}{2}\left(\frac{\partial b_{i}}{\partial x^{j}}+\frac{\partial b_{j}}{\partial x^{i}}\right)\right|_{o}=-\frac{1}{2}\left\langle\left[u_{i}, u_{j}\right]_{m}, u_{n}\right\rangle, i \geq j .
$$

Combining the above equation (3.15) with the connection coefficients $\Gamma_{i j}^{l}$ defined in [9], we get

Further

$$
r_{i j}(o)=-\frac{1}{2} c\left(\left\langle\left[u_{n}, u_{j}\right]_{m}, u_{i}\right\rangle+\left\langle\left[u_{n}, u_{i}\right]_{m}, u_{j}\right\rangle\right), i \geq j .
$$

$$
\left.r_{00}\right|_{o}=r_{i j}(o) y^{i} y^{j}=-c\left\langle\left[u_{n}, y\right]_{m}, y\right\rangle .
$$

Now by substituting the above quantities and the values of (3.3) and (3.4) into the equation (3.2) we get the formula for S-curvature of homogeneous Finsler space with the special $(\alpha, \beta)$-metric $F=\frac{\alpha^{2}}{\beta}+\beta$ at the origin as follows,

$$
S(o, y)=\frac{c(n+1) s}{b^{2} \alpha(y)\left(1+s^{2}\right)}\left\{c\left\langle\left[u_{n}, y\right]_{m}, y\right\rangle+\frac{\alpha(y)\left(s^{2}-1\right)}{2 s}\left\langle[u, y]_{m}, u\right\rangle,\right.
$$

Summarizing above statements we get,

Theorem 3.2. Let $F=\phi(s)$ be a G-invariant $(\alpha, \beta)$-metric on the reductive homogeneous manifold $G / H$, with a decomposition of the Lie algebra

Then the $S$-curvature of $F=\frac{\alpha^{2}}{\beta}+\beta$ has the form

$$
S(o, y)=\frac{c(n+1) s}{b^{2} \alpha(y)\left(1+s^{2}\right)}\left\{c\left\langle\left[u_{n}, y\right]_{m}, y\right\rangle+\frac{\alpha(y)\left(s^{2}-1\right)}{2 s}\left\langle[u, y]_{m}, u\right\rangle\right.
$$

where $u$ is the vector in $m$ corresponding to the 1-form $\beta$, and we have identified $m$ with the tangent space of $G / H$ at the origin $o=H$.

As a direct application of the above formula we get,

Theorem 3.3. Let $(G / H, F)$ be as in the above theorem (3.2). Then homogeneous Finsler space with special $(\alpha, \beta)$-metric $F$ has isotropic $S$-curvature if and only if $F$ has vanishing $S$-curvature. 
Proof: By using the formula derived in the previous theorem (3.2), it is enough to show the direct part. So, suppose $F$ has isotropic $S$-curvature;

$$
S(x, y)=(n+1) c(x) F(y), \quad x \in \frac{G}{H}, \quad y \in T_{x}(G / H) .
$$

Letting $x=0$ and $y=u$ then by (3.20) we get $c(0)=0$. Hence $S(o, y)=0, \forall y \in T o(G / H)$.

Since $F$ is a homogeneous metric, we must have $S=0$ everywhere.

\section{Mean Berwald Curvature Of Homogeneous Finsler Space}

In Finsler geometry, similar to the $S$-curvature, one more important non-Riemannian quantity is meanBerwald curvature. In this section by using the results of Section 3, we give a formula of mean Berwald curvature of homogeneous Finsler space with special $(\alpha, \beta)$-metric $F=\frac{\alpha^{2}}{\beta}+\beta$. In [5], X. Cheng and Z. Shen, give the mean Berwald curvature for Finsler metrics as follows,

$$
E_{i j}=\frac{1}{2} \frac{\partial^{2}}{\partial y^{i} \partial y^{j}}\left(\frac{\partial G^{m}}{\partial y^{m}}\right) \text {, }
$$

where $G^{m}=G^{m}(x, y)$ are the spray coefficients. By (2.10), $S$-curvature can be defined as

$$
S=\frac{\partial G^{m}}{\partial y^{m}}-(\ln \sigma(x))_{x^{k}} y^{k} .
$$

Here, since $\sigma(x)$ is the function of $x$, the second term in the above equation $(\ln \sigma(x))$ is also function of $\mathrm{x}$ only. Therefore,

$$
\frac{\partial^{2}}{\partial y^{i} \partial y^{j}}\left[(\ln \sigma(x))_{x^{k}} y^{k}\right]=0 .
$$

Hence, the formula for mean Berwald curvature obtained by direct computation,

$$
\begin{aligned}
\frac{\partial^{2} S}{\partial y^{i} \partial y^{j}} & =\frac{\partial^{2}}{\partial y^{i} \partial y^{j}}\left[\frac{\partial G^{m}}{\partial y^{m}}-(\ln \sigma(x))_{x^{k}} y^{k}\right] \\
& =\frac{\partial^{2}}{\partial y^{i} \partial y^{j}}\left(\frac{\partial G^{m}}{\partial y^{m}}\right)=2 \mathrm{E}_{\mathrm{ij}} .
\end{aligned}
$$

So that, clearly by partially differentiating the expression of $S$-curvature derived in section 3 , we can determine the formula for mean Berwald curvature of the homogeneous Finsler space. i.e.,

$$
2 \mathrm{E}_{\mathrm{ij}}=\frac{\partial^{2} S}{\partial y^{i} \partial y^{j}}
$$

Before the computation, we consider the identities,

$$
\frac{\partial^{2} s}{\partial y^{m}}=\frac{1}{\alpha}\left(b_{m}-s \frac{y^{m}}{\alpha}\right), \frac{\partial \alpha}{\partial y^{m}}=\frac{y^{m}}{\alpha}
$$

Theorem 4.4. Let $\left(\frac{G}{H}, F\right)$ be a homogeneous Finsler space with the special $(\alpha, \beta)$-metric of the form $F=\frac{\alpha^{2}}{\beta}+$ $\beta$, where $\alpha$ be a G-invariant Riemannian metric on $\frac{G}{H}$ and $\beta$ be a $G$-invariant vector field on $G / H$. Then the mean Berwald curvature of homogeneous Finsler space is of the form

$$
\begin{aligned}
E_{i j}=\frac{n+1}{b^{2}} & \left\{\frac{s}{\alpha(y)\left(1+s^{2}\right)}\left\langle\left[u, u_{i}\right]_{m}, u_{j}\right\rangle+\left\langle\left[u, u_{j}\right]_{m}, u_{i}\right\rangle+\left\langle[u, y]_{m}, y\right\rangle \frac{A}{\alpha^{5}\left(1+s^{2}\right)^{3}}\right. \\
& \left.+\left\langle[u, y]_{m}, u\right\rangle \frac{1}{2} \frac{B}{\alpha^{3}\left(1+s^{2}\right)^{3}}\right\}
\end{aligned}
$$

where $A$ and $B$ are defined in equations (4.17) and (4.18) respectively.

Proof: Let us consider Finsler $(\alpha, \beta)$-metric $F=\phi(s)$, where $\phi(s)=\frac{1}{s}+s$. Put $\psi=\phi-s \phi$, then we have,

$$
\begin{aligned}
& Q=\frac{\phi^{\prime}}{\psi}=-\frac{s^{2}-1}{2 s}, Q^{\prime}=\frac{s^{2}+1}{2 s^{2}}, \Delta=1+s Q+\left(b^{2}-s^{2}\right) Q^{\prime}=\frac{b^{2}\left(s^{2}+1\right)}{2 s^{2}} \\
& \Phi=-\left(Q-s Q^{\prime}\right) n \Delta+1+s Q-\left(b^{2}-s^{2}\right)(1+s Q) Q^{\prime \prime}=\frac{(n+1) b^{2}\left(1+s^{2}\right)}{2 s^{3}}
\end{aligned}
$$

Letting $c=1$ in the formula of S-curvature defined in equation (3.20), we get

Now by using (4.4) we obtain,

$$
\begin{aligned}
S(o, y) & =\frac{(n+1) s}{b^{2} \alpha(y)\left(1+s^{2}\right)}\left\{\left\langle[u, y]_{m}, y\right\rangle+\frac{\alpha(y)\left(s^{2}-1\right)}{2 s}\left\langle[u, y]_{m}, u\right\rangle\right\} \\
& =\frac{(n+1)}{b^{2}}\left\{\frac{s}{\alpha(y)\left(1+s^{2}\right)}\left\langle[u, y]_{m}, y\right\rangle+\frac{\left(s^{2}-1\right)}{2\left(1+s^{2}\right)}\left\langle[u, y]_{m}, u\right\rangle\right\}
\end{aligned}
$$

$$
2 E_{i j}=\frac{\partial^{2} S(o, y)}{\partial y^{i} \partial y^{j}}
$$


Now consider the first term,

$$
\begin{aligned}
= & \frac{(n+1)}{b^{2} \alpha(y)} \frac{\partial^{2}}{\partial y^{i} \partial y^{j}}\left\{\frac{s}{\left(1+s^{2}\right)}\left\langle[u, y]_{m}, y\right\rangle+\frac{\left(s^{2}-1\right)}{2\left(1+s^{2}\right)}\left\langle[u, y]_{m}, u\right\rangle\right\} \\
= & \frac{(n+1)}{b^{2}} \frac{\partial^{2}}{\partial y^{i} \partial y^{j}}\left\{\frac{s}{\alpha(y)\left(1+s^{2}\right)}\left\langle[u, y]_{m}, y\right\rangle+\frac{\left(s^{2}-1\right)}{2\left(1+s^{2}\right)}\left\langle[u, y]_{m}, u\right\rangle\right\} \\
= & \frac{(n+1)}{b^{2}}\left\{\frac{\partial^{2}}{\partial y^{i} \partial y^{j}}\left(\frac{s\left\langle[u, y]_{m}, y\right\rangle}{\alpha(y)\left(1+s^{2}\right)}\right)+\frac{\partial^{2}}{\partial y^{i} \partial y^{j}}\left(\frac{\left(s^{2}-1\right)\left\langle[u, y]_{m}, u\right\rangle}{2\left(1+s^{2}\right)}\right)\right\}
\end{aligned}
$$

$$
\begin{aligned}
& \frac{\partial^{2}}{\partial y^{i} \partial y^{j}}\left(\frac{s\left\langle[u, y]_{m}, y\right\rangle}{\alpha(y)\left(1+s^{2}\right)}\right)=\frac{s}{\alpha(y)\left(1+s^{2}\right)} \frac{\partial^{2}}{\partial y^{i} \partial y^{j}}\left(\left\langle[u, y]_{m}, y\right\rangle\right) \\
&+\left\langle[u, y]_{m}, y\right\rangle \frac{\partial^{2}}{\partial y^{i} \partial y^{j}}\left(\frac{s}{\alpha(y)\left(1+s^{2}\right)}\right)
\end{aligned}
$$

but we get,

$$
\begin{aligned}
& \frac{\partial^{2}\left\langle[u, y]_{m}, y\right\rangle}{\partial y^{i}}=\left\langle\left[u, u_{i}\right]_{m}, y\right\rangle+\left\langle[u, y]_{m}, u_{i}\right\rangle, \\
& \frac{\partial^{2}\left\langle[u, y]_{m}, y\right\rangle}{\partial y^{i} \partial y^{j}}=\left\langle\left[u, u_{i}\right]_{m}, u_{j}\right\rangle+\left\langle\left[u, u_{j}\right]_{m}, u_{i}\right\rangle
\end{aligned}
$$

where,

$$
\begin{aligned}
A & =\left[8 s^{2}\left(1+s^{2}\right) \alpha^{3}-\alpha^{3}\left(1+s^{2}\right)^{2}-\alpha^{2}\left(1+s^{2}\right)^{2}+2 s^{2} \alpha^{2}\left(1+s^{2}\right)-8 s^{4} \alpha^{3}\right]\left(b_{i}-\frac{s y_{i}}{\alpha}\right) y^{j} \\
& -10 b_{j} \alpha^{4} s\left(1+s^{2}\right)\left(b_{i}-\frac{s y_{i}}{\alpha}\right)-(1+\alpha) \alpha^{2} s\left(1+s^{2}\right)^{2} \delta_{i}^{j}-b_{j} \alpha^{2} y^{i}\left(1+s^{2}\right)^{2} \\
& +\left[2 s \alpha\left(1+s^{2}\right)+3\left(1+s^{2}\right)-4 s^{3} \alpha\right]\left(1+s^{2}\right) y^{i} y^{j}+2 b_{j} s^{2} \alpha^{2}\left(1+s^{2}\right) y^{i} \\
& +8 b_{j} s^{3} \alpha^{4}\left(b_{i}-\frac{s y_{i}}{\alpha}\right)+2 \alpha^{3} s^{3}\left(1+s^{2}\right) \delta_{i}^{j} .
\end{aligned}
$$

Therefore we have,

$$
\begin{aligned}
\frac{\partial^{2}}{\partial y^{i} \partial y^{j}}\left(\frac{s\left\langle[u, y]_{m}, y\right\rangle}{\alpha(y)\left(1+s^{2}\right)}\right)= & \frac{s}{\alpha(y)\left(1+s^{2}\right)}\left[\left\langle\left[u, u_{i}\right]_{m}, u_{j}\right\rangle+\left\langle\left[u, u_{j}\right]_{m}, u_{i}\right\rangle\right] \\
& +\left\langle[u, y]_{m}, y\right\rangle \frac{A}{\alpha^{5} s^{3}\left(1+s^{2}\right)^{3}}
\end{aligned}
$$

where $A$ is defined in the above equation (4.14). Similarly, by direct computation the value of second term of the equation (4.10) becomes,

where

$$
\frac{\partial^{2}}{\partial y^{i} \partial y^{j}}\left(\frac{\left(s^{2}-1\right)\left\langle[u, y]_{m}, u\right\rangle}{2\left(1+s^{2}\right)}\right)=\left\langle[u, y]_{m}, u\right\rangle \frac{B}{\alpha^{3}\left(1+s^{2}\right)^{3}}
$$

$$
\begin{aligned}
B=4 & {\left[\left(1+s^{2}\right)-4 s^{2}\right] b_{j} \alpha^{3}\left(b_{i}-\frac{s y^{i}}{\alpha}\right)+8 s\left(s^{2}-1\right) \alpha^{2}\left(b_{i}-\frac{s y_{i}}{\alpha}\right) y_{j} } \\
& -4 \alpha^{2} s^{2}\left(1+s^{2}\right) \delta_{i}^{j}+4 s^{2}\left(1+s^{2}\right) y^{i} y^{j} .
\end{aligned}
$$

Hence by substituting equations (4.15), (4.16) into (4.10) we get,

where,

$$
\begin{aligned}
E_{i j}=\frac{n+1}{b^{2}} & \left\{\frac{s}{\alpha(y)\left(1+s^{2}\right)}\left\langle\left[u, u_{i}\right]_{m}, u_{j}\right\rangle+\left\langle\left[u, u_{j}\right]_{m}, u_{i}\right\rangle+\left\langle[u, y]_{m}, y\right\rangle \frac{A}{\alpha^{5}\left(1+s^{2}\right)^{3}}\right. \\
& \left.+\left\langle[u, y]_{m}, u\right\rangle \frac{1}{2} \frac{B}{\alpha^{3}\left(1+s^{2}\right)^{3}}\right\}
\end{aligned}
$$

$$
\begin{aligned}
A= & {\left[8 s^{2}\left(1+s^{2}\right) \alpha^{3}-\alpha^{3}\left(1+s^{2}\right)^{2}-\alpha^{2}\left(1+s^{2}\right)^{2}+2 s^{2} \alpha^{2}\left(1+s^{2}\right)-8 s^{4} \alpha^{3}\right]\left(b_{i}-\frac{s y_{i}}{\alpha}\right) y^{j} } \\
& -10 b_{j} \alpha^{4} s\left(1+s^{2}\right)\left(b_{i}-\frac{s y_{i}}{\alpha}\right)-(1+\alpha) \alpha^{2} s\left(1+s^{2}\right)^{2} \delta_{i}^{j}-b_{j} \alpha^{2} y^{i}\left(1+s^{2}\right)^{2} \\
& +\left[2 s \alpha\left(1+s^{2}\right)+3\left(1+s^{2}\right)-4 s^{3} \alpha\right]\left(1+s^{2}\right) y^{i} y^{j}+2 b_{j} s^{2} \alpha^{2}\left(1+s^{2}\right) y^{i} \\
& +8 b_{j} s^{3} \alpha^{4}\left(b_{i}-\frac{s y_{i}}{\alpha}\right)+2 \alpha^{3} s^{3}\left(1+s^{2}\right) \delta_{i}^{j} . \\
B= & 4\left[\left(1+s^{2}\right)-4 s^{2}\right] b_{j} \alpha^{3}\left(b_{i}-\frac{s y^{i}}{\alpha}\right)+8 s\left(s^{2}-1\right) \alpha^{2}\left(b_{i}-\frac{s y_{i}}{\alpha}\right) y_{j} \\
& -4 \alpha^{2} s^{2}\left(1+s^{2}\right) \delta_{i}^{j}+4 s^{2}\left(1+s^{2}\right) y^{i} y^{j} .
\end{aligned}
$$

This is the required formula of mean Berwald curvature of homogeneous Finsler space. This completes the proof. 


\section{Conclusion}

$S$-curvature is an important geometric quantity in Finsler geometry in that it has some mysterious interrelations with other quantities such as flag curvature, Ricci scalar, etc. Shen showed that the BishopGromov volume comparison theorem holds for Finsler spaces with vanishing $S$-curvature. Therefore, it is also significant to characterize homogeneous Finsler spaces with vanishing $S$-curvature. In this paper, we derived an explicit formula of the $S$-curvature of homogeneous Finsler space with special $(\alpha, \beta)$-metric and proved that a homogeneous Finsler space with almost isotropic $S$-curvature must have vanishing S-curvature.

We also derived an explicit formula of the mean Berwald curvature $E_{i j}$ of homogeneous Finsler space.

\section{References}

[1]. S. Bacso, X. Cheng and Z. Shen., Curvature Properties of $(\alpha, \beta)$-metrics, Adv. Stud. Pure Math. Soc., Japan (2007).

[2]. K. Chandru and S. K. Narasimhamurthy, On curvatures of homogeneous Finsler-Kropina space, Gulf Journal of Mathematics Vol 5, Issue 1 (2017) 73-83.

[3]. K. Chandru, S. K. Narasimhamurthy and M. Ramesha, A study of nonholonomic Finsler frame on gauge transformation, Bulletin of the Transilvania University of Brasov, Vol 9(58), No. 1-2016, Series III: Mathematics, Informatics, Physics, 29-38.

[4]. S.S. Chern, Z. Shen, RiemannFinsler Geometry, World Scientific Publishers, (2004).

[5]. X. Cheng and Z. Shen, A class of Finsler metrics with isotropic S-curvature, Israel J. Math. 169, 317-340, (2009),

[6]. S. Deng and Z. Hou, The group of isometries of a Finsler space, Pac. J. Math. 207, 149-157, (2002).

[7]. S. Deng and X. Wang, The S-curvature of homogeneous $(\alpha, \beta)$--metrics, Balkan Journal of Geometry and Its Applications, Vol.15, No.2, pp. 39-48, (2010).

[8]. S. Deng and Z. Hou, Homogeneous Finsler spaces of negative curvature, Journal of Geometry and Physics, 57, 657-664, (2007).

[9]. Z. Hu and S. Deng, Three dimensional homogeneous Finsler manifolds, Math. Nachr. 285, No. 10, 1243 1254 (2012)

[10]. S. Kobayashi and K. Nomizu, Foundations of Differential Geomtry, Interscience Pub- lishers, Vol. 1, 1963; Vol. 2, (1969).

[11]. V. K. Kropina, On projective Finsler spaces with a certain special form, Naucn. Doklady vyss. Skoly, fiz.-mat. Nauki, 19-59(2), 3842, Russian, (1960).

[12]. Shaoqiang Deng, The S-curvature of homogeneous Randers spaces, Differential Geometry and its Applications, 27, 75-84,(2009).

[13]. C. Shibata, On Finsler spaces with Kropina metric, Rep. Math. Phys., 13, 117-128, (1978).

[14]. Z. Shen, Volume comparison and its applications in Riemann Finsler geometry, Adv. Math. 128, 306-328, (1997). 